\title{
Altered pituitary morphology as a sign of benign hereditary chorea caused by TITF1/NKX2.1 mutations
}

\author{
Steffi Thust ${ }^{1} \cdot$ Liana Veneziano $^{2} \cdot$ Michael H. Parkinson $^{3} \cdot$ Kailash P. Bhatia $^{4} \cdot$ Elide Mantuano $^{2}$. \\ Cristina Gonzalez-Robles ${ }^{3} \cdot$ Indran Davagnanam ${ }^{5} \cdot$ Paola Giunti $^{3}$ (i)
}

Received: 25 September 2021 / Accepted: 23 December 2021 / Published online: 25 January 2022

(c) The Author(s) 2022

\begin{abstract}
Benign hereditary chorea $(\mathrm{BHC})$ is a rare genetically heterogeneous movement disorder, in which conventional neuroimaging has been reported as normal in most cases. Cystic pituitary abnormalities and features of empty sella have been described in only 7 patients with BHC to date. We present 4 patients from 2 families with a BHC phenotype, 3 of whom underwent targeted pituitary MR imaging and genetic testing. All four patients in the two families displayed a classic BHC phenotype. The targeted pituitary MR imaging demonstrated abnormal pituitary sella morphology. Genetic testing was performed in three patients, and showed mutations causing BHC in three of the patients, as well as identifying a novel nonsense mutation of the TITF1/NKX2-1 gene in one of the patients. The presence of the abnormal pituitary sella in two affected members of the same family supports the hypothesis that this sign is a distinct feature of the BHC phenotype spectrum due to mutations in the TITF1 gene. Interestingly, these abnormalities seem to develop in adult life and are progressive. They occur in at least $26 \%$ of patients affected with Brain-lung-thyroid syndrome. As a part of the management of these patients we recommend to perform follow-up MRI brain with dedicated pituitary imaging also in adult life as the abnormality can occur years after the onset of chorea.
\end{abstract}

Keywords Benign hereditary chorea $\cdot$ Brain-lung-thyroid syndrome $\cdot$ Pituitary gland $\cdot$ Pituitary cyst $\cdot$ NKX2.1

\section{Abbreviations \\ ADCY5 Adenylate cyclase 5 \\ BHC Benign hereditary chorea \\ BLT Brain-lung-thyroid (syndrome)}

Indran Davagnanam

indran.davagnanam@nhs.net

$\triangle$ Paola Giunti

p.giunti@ucl.ac.uk

1 National Hospital for Neurology and Neurosurgery, Queen Square, London WC1N 3BG, UK

2 Institute of Translational Pharmacology, National Research Council of Italy, Via Fosso del Cavaliere 100, 00133 Rome, Italy

3 Ataxia Centre, Department of Clinical and Motor Neuroscience, UCL Queen Square Institute of Neurology, London WC1N 3BG, UK

4 Department of Clinical and Motor Neuroscience, UCL Institute of Neurology, QueenSquare, London WC1N 3BG, UK

5 Brain Repair and Rehabilitation Unit, UCL Institute of Neurology, QueenSquare, London WC1N 3BG, UK

$\begin{array}{ll}\text { cAMP } & \text { Cyclic adenosine monophosphate } \\ \text { cGMP } & \text { Cyclic guanosine monophosphate } \\ \text { CSF } & \text { Cerebrospinal fluid } \\ \text { CT } & \text { Computerized tomography } \\ \text { HGVS } & \text { Human Genome Variation Society } \\ \text { MRI } & \text { Magnetic resonance imaging } \\ \text { MSH } & \text { Melanocyte-stimulating hormone } \\ \text { NKX2-1 } & \text { NK2 Homeobox 1 } \\ \text { PDE10A } & \text { Phosphodiesterase 10A } \\ \text { T/EBP } & \text { Thyroid-specific enhancer-binding protein } \\ \text { TITF-1 } & \text { Thyroid transcription factor 1 }\end{array}$

\section{Introduction}

Benign hereditary chorea (OMIM: \#118700) is a rare possibly dominantly inherited hyperkinetic movement disorder, with underlying heterogeneous genetic causes. The first gene that has been identified is NKX2-1, also known as TITF-1, on chromosome 14q13.3 coding for the thyroid transcription factor 1 [1]. Mutations in a second gene, ADCY5, coding for the adenylate cyclase 5 , have been found to be another 
cause of benign hereditary chorea [2]. Recently, mutations in PDE10A, encoding an enzyme involved in the hydrolysis/degradation of cAMP and cyclic guanosine monophosphate (cGMP), have been reported in patients with infantile/ childhood-onset chorea [3]. TITF1/NKX2-1 encodes for a thyroid-specific enhancer-binding protein $(T / E B P)$, which plays a regulatory role in thyroid, brain, and lung organogenesis. Hence, the underlying genetic defect may manifest as "Brain-Lung-Thyroid syndrome" (BLT, OMIM: \#610978), characterized by a broad phenotypical spectrum, including neurological abnormalities, congenital hypothyroidism, infant respiratory distress syndrome, recurrent pulmonary infections, or interstitial lung disease [4-6]. Several TITF1/ NKX2-1 mutations have been identified, in which brain MR imaging was unremarkable. However, the presence of pituitary abnormalities has been described in a small proportion of BHC patients, 7 to date [7-10] This subset of patients raised the question of whether these findings were incidental or represented part of the variable BHC phenotype.

\section{TITF1 in pituitary development}

The pituitary gland originates from two embryonic tissues: the oral ectoderm for the adenohypophysis (the anterior and intermediate lobes) and the neural ectoderm for the neurohypophysis (the posterior lobe).

The anterior, intermediate, and posterior lobes of the pituitary gland function as three separate endocrine organs, each characterized by distinct cell populations, secretory products, and regulatory mechanisms.

The anterior lobe is a highly specialized tissue that contains a functionally diverse population of cell types committed to synthesize and secrete five different hormones during development [1]. The intermediate lobe is rudimentary in humans but produces MSH. Pituitary cysts are generally located in this portion. The posterior lobe releases oxytocin and vasopressin from axon terminals that originate in cell bodies located in the hypothalamus [11]. Pituitary development occurs in successive steps that are controlled by several transcription factors having a distinct temporal and spatial expression pattern. They interact with each other and with additional exogenous and endogenous signals to control cell determination and differentiation [11]. TITF1 is one of the numerous transcription factors involved in the development of the pituitary and acts at a very early stage, during the formation of the posterior lobe. In spite of TITF1 not being expressed in the intermediate and anterior lobe, in the TITF1 null mouse, the pituitary is completely missing, suggesting that the presence of the posterior lobe and/or TITF1 gene expression is required for full development of the anterior and intermediate pituitary [11-13]. Interestingly, TITF1 plays a pleiotropic function having various roles in different stages of the development and differentiation of several organs, such as lung, brain, thyroid, and pituitary. The pleiotropic functions are due to the action of two different activation domains and to specific post-translational modifications. [12]

Here, we present pituitary imaging in three patients, in whom recently a novel TITF1 mutation was discovered and all of which had an altered sella morphology. [8]

This work was approved by REC 04/Q0505/21.

\section{Clinical features}

The neurological presentation of BHC is typically in childhood before the age of 5 years, although age of onset may be variable from infancy to adolescence $[14,15]$. Typical signs include early hypotonia and delayed motor development, followed by walking difficulties, ataxia, with frequent falls, and usually later onset of chorea [16, 17]. Typically, cognition and speech are preserved, although cases of cognitive impairment and even psychiatric disturbance have been reported [18-21]. A progressive course in BHC is rare, although this has been described, and life expectancy lies within the normal range [22,23]. Despite the original name "benign hereditary chorea," only $13 \%$ presented with isolated chorea [24]. Some patients exhibit dystonia, myoclonus, tremor, ataxia, and dysarthria, which can make the clinical distinction between BHC and other neurological syndromes challenging [25-27]. The 50\% of patients with NKX2-1 mutations presented with a combination of neurological, pulmonary, and thyroid symptomatology. Thus, the "benign" phenotype initially described is actually uncommon [28]. In light of the varied manifestations of heterozygous mutations in NKX2-1, some authors suggest that the term hereditary benign chorea should be replaced by $N K X 2$ 1-related disorders. [29]

\section{Materials and methods}

\section{Family 1}

This 49-year-old subject presented in infancy with delayed milestones before being diagnosed with cerebellar ataxia at the age of 2 . The patient had lifelong balance problems with onset of jerky movements in adolescence. Over the last 10 years, some worsening in choreiform movements was noticed, as well as an increased frequency of falls. Physical examination revealed mild gait ataxia with dystonic posturing of the hands, choreic jerks, and dystonic movements of the head and shoulders. Mild ocular apraxia was noted as well as a degree of dysdiadochokinesia due to intrusion of involuntary movements.

We also examined a 26-year-old patient who is the offspring of the above described subject (case 1). Similar to case 1 , delayed motor development was present, with 
independent walking achieved at the age of 2 . Balance problems and frequent falls were present throughout childhood despite receiving intensive physiotherapy, with mild spontaneous improvement in adulthood. Examination revealed choreiform movements of the head and legs with no other neurological abnormality.

There are no other affected family members.

\section{Family 2}

The third patient arrived to our clinics at the age of 35 . The medical history showed delayed motor milestones. At birth, twitching movements of the limbs spreading throughout the body were noticed. Ataxia and falls were common in childhood and slowly improved. On examination, gait was impaired by both chorea and dystonia. One of their children was also born prematurely, with difficulties in feeding and delayed motor milestones. The affected child continued to experience falls and problems with walking, and had similar examination findings to the affected parent.

The pedigree of both families is detailed in Supplementary Figure 1.

\section{Molecular genetics}

Screening for mutations in the NKX2.1 gene, genomic DNA was amplified by PCR using primer pairs as described by Breedveld et al [1]. The obtained DNA fragments, all the three NKX2.1 coding exons, were sequenced with the Sanger method by Eurofins Genomics service (https://eurof insgenomics.eu/en/eurofins-genomics-genomic-services-byexperts/).

Screening for mutations in the NKX2.1 gene was performed in all affected subjects since mutations of this gene are the main cause of BHC phenotype. No other gene was screened because the diagnostic workflow suggests to screen the NKX2.1 gene as the first step. Mutations of other genes, as ADCY5 and PDE10A, are rarer.

\section{Results}

\section{Family 1}

\section{Laboratory and genetic results}

The 49-year-old patient had hypothyroidism, but otherwise had normal pituitary function tests. A biochemical profile performed on the 26-year-old patient showed a mildly reduced prolactin level and marginally raised thyroid-stimulating hormone (normal free T4 level).

Both parent and child showed the heterozygous nucleotide substitution NM_001079668.2:c.631A>T, which results in the change of a lysine residue for a stop codon at position 211, NM_001079668.2(NKX2-1_i001):p. (Lys211*) (nomenclature according to HGVS format). This de novo mutation was previously reported by our group [8].

\section{Imaging results}

Targeted high-resolution pituitary MR imaging of the 49-year-old subject demonstrated marked expansion of the CSF-filled sella turcica (Figure 1) with a slender rim of pituitary gland tissue draped along the anterior wall and floor of the sella. Corresponding CT imaging revealed hinning of the pituitary fossa bone margins without evidence of bone destruction. The remaining midline brain structures were normal on imaging.

Whole brain MR imaging supplemented by dedicated thin-section $(3 \mathrm{~mm})$ pituitary views of the 26 -year-old patient showed marked cystic expansion of the sella turcica with mild anterior displacement of the pituitary stalk and gland (Figure 2).

\section{Family 2}

\section{Laboratory and genetic results}

Both parent and child carry a heterozygous mutation NM_001079668.2:c.605A $>\mathrm{G}$ which is predicted to change a glutamine to an arginine NM_001079668.2(NKX2-1_ i001):p.(Gln202Arg). This is an evolutionarily highly conserved residue, not reported in databases of polymorphic variants.

In silico analysis of the missense mutation was performed using VARSOME software (https://varsome.com/) which is an annotation tool and search engine for human genomic variants, and a platform enabling the sharing of knowledge on specific variants According to all the 30 databases involved in VARSOME evaluation, this mutation results likely pathogenic (https://varsome.com/variant/hg19/NKX2.1\%3AQ20 $2 \mathrm{R}$ ? annotation-mode $=$ germline) .

Furthermore, a functional characterization of a different mutation of the same amino acid (NM_001079668.2:c.606G >C; p.Gln202His) showed that it causes a decrease in the DNA-binding activity, leading to a loss of protein function [30].

\section{Imaging results}

The parent's MR imaging demonstrated subtly altered morphology of the intrasellar structures with cystic enlargement of the CSF space anterior to the pituitary gland and flattening of the adenohypophyseal surface (Figure 3). No other intracranial abnormality was present. 

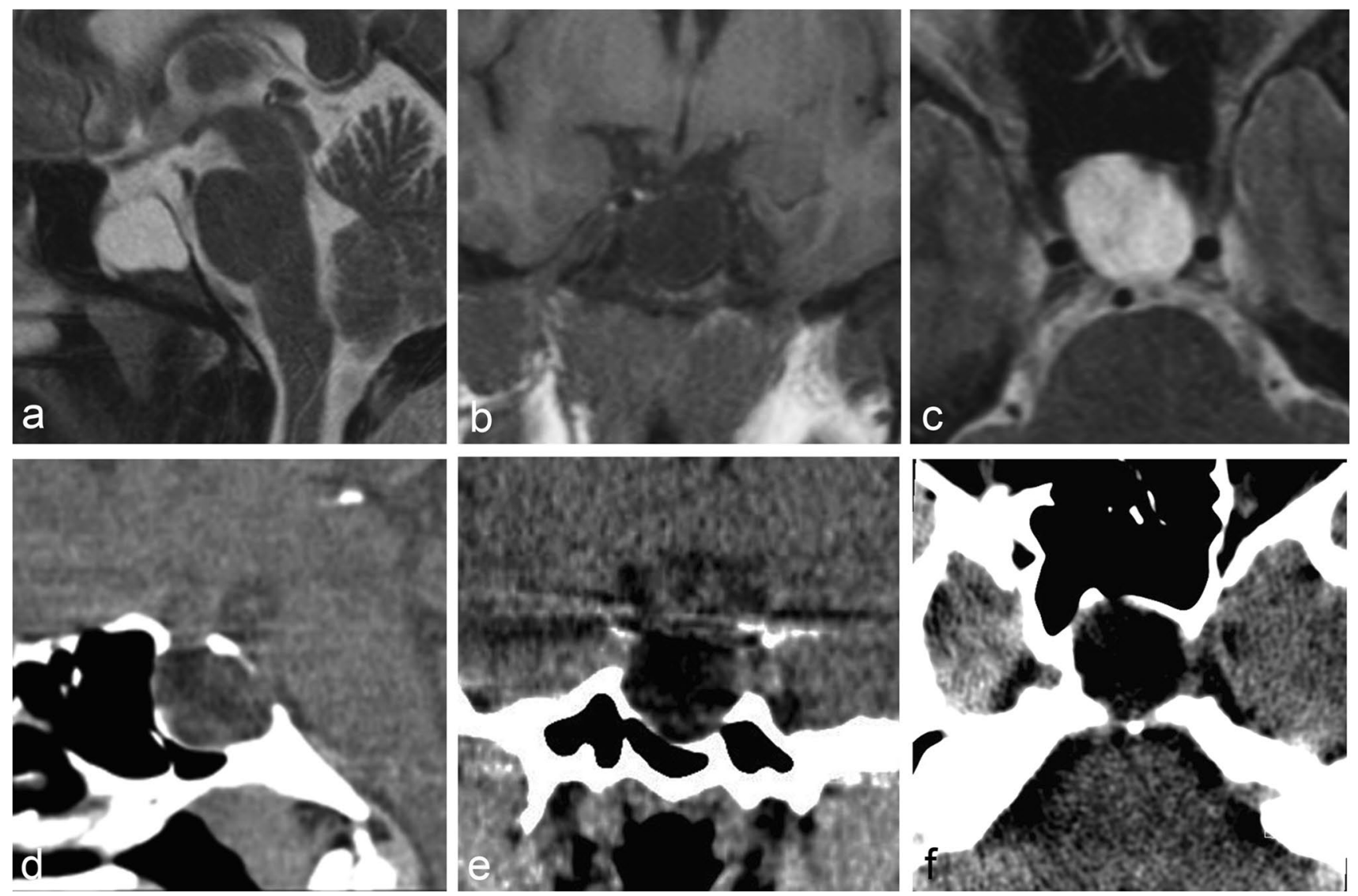

Figure 1: Non-contrast MRI (panels a-c) of the pituitary gland with sagittal (a) and axial (c) T2-weighted as well as coronal T1-weighted dedicated 3-mm thin-sections from case 1, demonstrating thin rim of pituitary tissue along the anterior and inferior aspects of the enlarged
CSF-filled sella turcica. Non-contrast multiplanar reformatted CT images (panels d-f) in the corresponding sagittal (d), coronal (e), and axial (f) planes demonstrating smooth remodelling of the osseous boundaries of the sella turcica with no evidence of bony erosion.
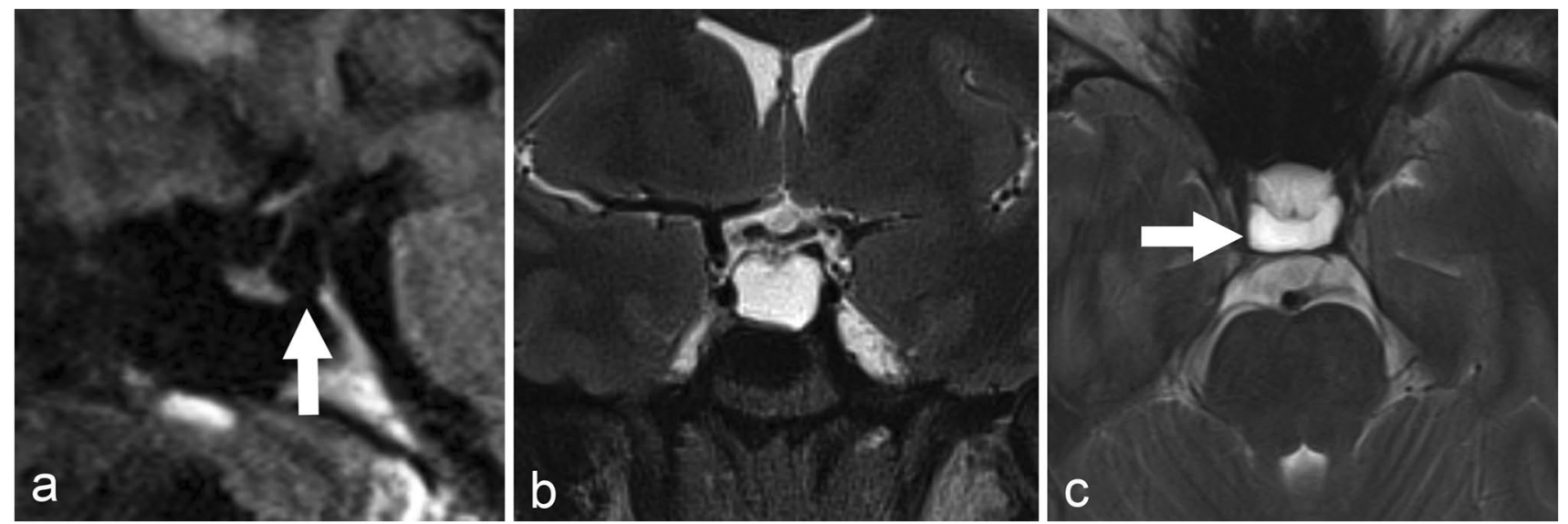

Figure 2: Non-contrast MRI (panels a-c) of the pituitary gland with sagittal (a) T1-weighted as well as coronal (b) and axial (c) T2-weighted dedicated 3-mm thin-sections from case 2 . The imaging demonstrates predominantly dorsal expansion of the intrasellar space (white arrow in panel a) with anterior deviation of the infundibulum and relative flattening of the superior contour of the pituitary gland. 

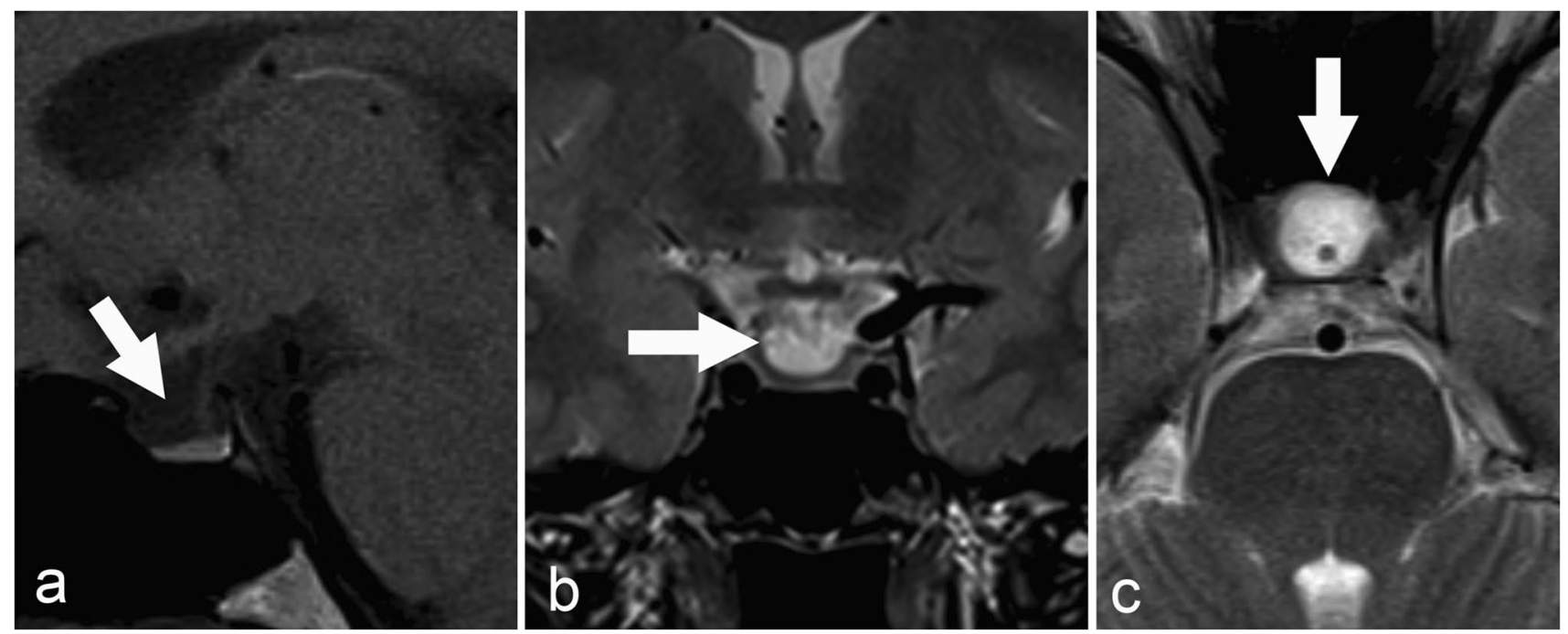

Figure 3: Non-contrast MRI (panels a-c) of the pituitary gland with sagittal (a) T1-weighted as well as coronal (b) and axial (c) T2-weighted dedicated 3-mm thin-sections from case 3. There is sub-

\section{Discussion}

The relationship of TITF-1 deficiency with abnormal basal ganglia development, in particular impaired striatal differentiation, has been well established in mice $[1,11]$. Loss of striatal interneurons was demonstrated in a human pathological specimen of a patient with BHC [31]. TITF-1 seems to play a critical role for the interneuron specification of medial ganglionic eminence cells [32], and the regulation of the direction of the migrating interneurons [33]. Importantly, TITF-1 has also been shown to promote development of the posterior pituitary and hypothalamus $[34,35]$. In fact, in a study by Kimura et al., homoygous TITF1 knockout mice were born dead and lacking lungs, thyroid, and pituitary gland. [11]

Krude et al. described two patients with a posterior pituitary cystic mass [7]. Accornero et al. presented a single case of a patient with pituitary stalk duplication and changes in the basal ganglia, caused by a deletion on chromosome 14 harboring TITF1. [35] Salvatore et al. identified features of "empty sella" in two adult patients, whereby the abnormality was more marked in the parent who had longer disease duration [5]. Balicza et al. reported a family where two patients with stop mutation of NKX2-1 gene had "empty sella" on MRI and pituitary hormone deficiencies [10]. The imaging features in the latter publication are strikingly similar to the patients in our series, supporting the hypothesis of progression over time. [8]

The three English cases presented here represent mutations in exon 3 of the TITF 1 gene encoding for the homeodomain of TTF-1, where most point mutations associated with BHC are located. All three patients demonstrate altered tle expansion of the anterior recess of the sella to thicker with flattening of the superior contour of the adenohypophysis (white arrows in panels a-c)

sella turcica morphology ranging from subtle flattening of the superior gland surface (case 2 and 3 ) to a large posterior intrasellar cyst (case 1). The mechanism for the development of these changes is unclear, but could represent a combination of congenital maldevelopment and acquired pathology, possibly as a result of local CSF pressure, given the presence of bony thinning in the oldest patient, who is most severely affected. Interestingly, one group described intrasellar cyst formation unrelated to $\mathrm{BHC}$ in the context of a persistent embryonal infundibular recess proven at surgery [36]. The exact mechanism for the development of sella abnormalities in BHC remains still unknown. Many mutations have been identified in TITF1 gene (large gene deletions and missense and nonsense mutations spanning the entire gene), but there is no relationship between the type of mutation and the severity of the phenotype. Clinical heterogeneity and incomplete penetrance of the disease cannot be predicted only on the basis of the mutation type. Environmental factors, tissue factors, and genetic background could influence the clinical phenotype of BHC patients [1]. Severity and organ involvement may also vary in a single pedigree [37]. Chorea can be the predominant or the only symptom associated with TITF1 gene mutations [25, 38]. With such clinical heterogeneity of the disease, we cannot exclude cases in which pituitary cysts or pituitary malformations could be the only symptom associated to TITF1 gene mutations. Therefore, it could be relevant looking for mutations in TITF1 gene in patients in which pituitary cysts or pituitary malformations have been diagnosed.

Among 98 cases of TITF1/NKX2-1 mutations published to date (see Table 1), most report normal imaging findings or do not feature imaging descriptions. A few groups 
Table 1. Mutations in TITF1/NKX2.1 with neurological, thyroid, lung, or pituitary involvement

\begin{tabular}{|c|c|c|c|c|c|c|c|c|c|}
\hline Mutation & $\begin{array}{l}\text { Transmis- } \\
\text { sion }\end{array}$ & Brain & Thyroid & Lung & Brain MRI & $\begin{array}{l}\text { N. patients } \\
\text { with brain } \\
\text { MRI }\end{array}$ & $\begin{array}{l}\text { N. patients } \\
\text { with pitui- } \\
\text { tary abnor- } \\
\text { malities }\end{array}$ & Notes & References \\
\hline §p.M59AfsX40 & De novo & + & - & + & NR & & & & $\begin{array}{l}\text { McMichael, } \\
2013 \text { [40] }\end{array}$ \\
\hline §p.Y98X & De novo & + & + & - & NR & & & & $\begin{array}{l}\text { Tübing, } 2018 \\
\text { [41] }\end{array}$ \\
\hline p.Y98X & $\mathrm{AD}$ & + & + & + & - & 2 & & & $\begin{array}{l}\text { Nakamura, } \\
2012 \text { [42] }\end{array}$ \\
\hline p.Q107X & $\mathrm{AD}$ & + & - & - & - & 4 & & & $\begin{array}{l}\text { Sempere, } 2013 \\
\text { [43] }\end{array}$ \\
\hline p.G115AfsX10 & De novo & + & - & + & - & 1 & & & $\begin{array}{l}\text { Parnes, } 2018 \\
\text { [28] }\end{array}$ \\
\hline p.Y116fsX323 & De novo & + & + & + & - & 1 & & & $\begin{array}{l}\text { Pohlenz, } 2002 \\
\text { [44] }\end{array}$ \\
\hline p.Y116X & $\mathrm{AD}$ & + & - & - & NR & & & & Gras, 2012 [45] \\
\hline p.C117X & $?$ & + & + & + & NR & & & & $\begin{array}{l}\text { Krude, } 2002 \\
\text { [7] }\end{array}$ \\
\hline p.P129fsX307 & De novo & + & - & + & NR & & & & $\begin{array}{l}\text { Hamvas, } 2013 \\
\text { [46] }\end{array}$ \\
\hline p.Y130X & De novo & + & + & + & - & 1 & & & $\begin{array}{l}\text { Parnes, } 2018 \\
\text { [28] }\end{array}$ \\
\hline p.Y130X & De novo & + & + & + & - & 1 & & & $\begin{array}{l}\text { Iodice, } 2019 \\
\text { [47] }\end{array}$ \\
\hline p.T133NfsX306 & De novo & - & + & + & - & 1 & & & $\begin{array}{l}\text { Parnes, } 2018 \\
\text { [28] }\end{array}$ \\
\hline §p.W143X & De novo & + & + & - & + & 2 & 2 & Empty sella & $\begin{array}{l}\text { Balicza, } 2018 \\
\text { [10] }\end{array}$ \\
\hline p.Y144X & $?$ & + & + & - & NR & & & & $\begin{array}{l}\text { Teissier, } 2012 \\
\text { [48] }\end{array}$ \\
\hline p.Y144X & $?$ & + & + & + & NR & & & & $\begin{array}{l}\text { Hamvas, } 2013 \\
\text { [46] }\end{array}$ \\
\hline §p.R157AfsX7 & De novo & + & + & - & - & 1 & & & Milone, 2019 [] \\
\hline c. $463+1 \_463$ + 4del & $\mathrm{AD}$ & + & + & - & NR & & & & Gras, 2012 [45] \\
\hline c. $463+1 \mathrm{G}>\mathrm{A}$ & De novo & + & + & + & - & 1 & & & $\begin{array}{l}\text { Fons, } 2012 \\
\text { [49] }\end{array}$ \\
\hline c. $464-9 \mathrm{C}>\mathrm{A}$ & $\mathrm{AD}$ & + & + & - & - & 6 & & & $\begin{array}{l}\text { Konishi, } 2013 \\
\text { [50] }\end{array}$ \\
\hline c. $464-1 \mathrm{G}>\mathrm{A}$ & De novo & + & + & + & - & 1 & & & $\begin{array}{l}\text { Barreiro, } 2011 \\
\text { [51] }\end{array}$ \\
\hline c. $464-2 \mathrm{~A}>\mathrm{C}$ & $\mathrm{AD}$ & + & - & - & - & 2 & & & $\begin{array}{l}\text { Asmus, } 2007 \\
{[25]}\end{array}$ \\
\hline c. $464-2 \mathrm{~A}>\mathrm{T}$ & $\mathrm{AD}$ & + & - & NR & - & 1 & & & $\begin{array}{l}\text { Kleiner- } \\
\text { Fisman,2003 } \\
\text { [31] }\end{array}$ \\
\hline c. $464-2 \mathrm{~A}>\mathrm{G}$ & $\mathrm{AD}$ & + & + & + & - & 2 & & & $\begin{array}{l}\text { Doyle, } 2004 \\
\text { [52] }\end{array}$ \\
\hline c. $464-2 \mathrm{~A}>\mathrm{G}$ & De novo & + & + & + & - & 1 & & & $\begin{array}{l}\text { Carrè, } 2009 \\
\text { [24] }\end{array}$ \\
\hline p.S163fsX2 & De novo & + & + & + & NR & & & & Gras, 2012 [45] \\
\hline p.S175X & $\mathrm{AD}$ & + & + & + & + & 2 & 2 & Empty sella & $\begin{array}{l}\text { Ferrara, } 2008 \\
\text { [53]; Salva- } \\
\text { tore, } 2010 \text { [5] }\end{array}$ \\
\hline p.P185fsX250 & De novo & + & + & + & NR & & & & $\begin{array}{l}\text { Hamvas, } 2013 \\
\text { [46] }\end{array}$ \\
\hline
\end{tabular}


Table 1. (continued)

\begin{tabular}{|c|c|c|c|c|c|c|c|c|c|}
\hline Mutation & $\begin{array}{l}\text { Transmis- } \\
\text { sion }\end{array}$ & Brain & Thyroid & Lung & Brain MRI & $\begin{array}{l}\text { N. patients } \\
\text { with brain } \\
\text { MRI }\end{array}$ & $\begin{array}{l}\text { N. patients } \\
\text { with pitui- } \\
\text { tary abnor- } \\
\text { malities }\end{array}$ & Notes & References \\
\hline p.P187fsX196 & De novo & + & + & + & - & 1 & & & $\begin{array}{l}\text { Nagasaki, } 2008 \\
\text { [54] }\end{array}$ \\
\hline p.R195fsX32 & $\mathrm{AD}$ & + & + & + & - & 1 & & & $\begin{array}{l}\text { Nettore, } 2013 \\
\text { [55] }\end{array}$ \\
\hline p.R195W & De novo & + & + & + & NR & & & & $\begin{array}{l}\text { Hamvas, } 2013 \\
\text { [46] }\end{array}$ \\
\hline p.L197P & $?$ & + & - & + & NR & & & & $\begin{array}{l}\text { Hamvas, } 2013 \\
\text { [46] }\end{array}$ \\
\hline p.F198L & $\mathrm{AD}$ & - & - & + & NR & & & & $\begin{array}{l}\text { Hamvas, } 2013 \\
\text { [46] }\end{array}$ \\
\hline p.F198L & $?$ & - & - & + & NR & & & & $\begin{array}{l}\text { Hamvas, } 2013 \\
\text { [46] }\end{array}$ \\
\hline p.F198L & $?$ & - & - & + & NR & & & & $\begin{array}{l}\text { Hamvas, } 2013 \\
\text { [46] }\end{array}$ \\
\hline p.S199X & $?$ & + & + & - & NR & & & & $\begin{array}{l}\text { Krude, } 2002 \\
\text { [7] }\end{array}$ \\
\hline §p.Q202H & De novo & + & + & - & - & & & & $\begin{array}{c}\text { Provenzano, } \\
2016[30]\end{array}$ \\
\hline p.Q202R & De novo & + & - & - & + & 1 & 1 & Empty sella & Present paper \\
\hline p.E205X & $\mathrm{AD}$ & - & - & - & - & 3 & & & $\begin{array}{l}\text { Asmus, } 2005 \\
\text { [56] }\end{array}$ \\
\hline p.L206V & De novo & + & - & - & - & 1 & & & $\begin{array}{c}\text { Gras, } 2012 \\
\text { [45]; Carrè, } \\
2009 \text { [24] }\end{array}$ \\
\hline p.R208X & $\mathrm{AD}$ & + & - & - & - & 1 & & & $\begin{array}{c}\text { Provenzano, } \\
2008 \text { [57] }\end{array}$ \\
\hline p.R209P & De novo & + & + & - & - & 2 & & & $\begin{array}{c}\text { Williamson, } \\
2014 \text { [58] }\end{array}$ \\
\hline p.K211X & De novo & + & + & - & + & 2 & 2 & $\begin{array}{l}\text { Cystic mass, } \\
\text { empty sella }\end{array}$ & $\begin{array}{c}\text { Veneziano, } \\
2014 \text { [8] }\end{array}$ \\
\hline p.Y215D & De novo & + & + & - & NR & & & & Gras, 2012 [45] \\
\hline p.S217X & $\mathrm{AD}$ & + & + & - & NR & & & & Glik, 2008 [20] \\
\hline p.L224R & $\mathrm{AD}$ & + & + & - & NR & & & & Gras, 2012 [45] \\
\hline p.L224R & De novo & + & + & + & NR & & & & $\begin{array}{l}\text { Koht, } 2016 \\
\text { [59] }\end{array}$ \\
\hline p.A225fsX228 & De novo & + & - & - & NR & & & & $\begin{array}{l}\text { Krude, } 2002 \\
\text { [7] }\end{array}$ \\
\hline p.L230P & De novo & + & + & - & - & 1 & & & $\begin{array}{l}\text { Iodice, } 2019 \\
\text { [47] }\end{array}$ \\
\hline p.P233L & De novo & + & + & - & NR & & & & $\begin{array}{l}\text { Carrè, } 2009 \\
\text { [24] }\end{array}$ \\
\hline p.V235P & De novo & + & + & + & + & 1 & 1 & Cystic mass & $\begin{array}{l}\text { Krude, } 2002 \\
\text { [7] }\end{array}$ \\
\hline p.V235P & $\mathrm{AD}$ & + & + & - & - & 2 & & & $\begin{array}{l}\text { Uematsu, } 2012 \\
\text { [18] }\end{array}$ \\
\hline p.I237F & De novo & NR & + & + & NR & & & & $\begin{array}{l}\text { Maquet, } 2009 \\
\text { [60] }\end{array}$ \\
\hline p.I237M & De novo & - & + & + & NR & & & & $\begin{array}{l}\text { Gillet, } 2013 \\
\text { [61] }\end{array}$ \\
\hline p.W238L & $\mathrm{AD}$ & + & NR & NR & - & 1 & & & $\begin{array}{l}\text { Breedveld, } \\
2002[1]\end{array}$ \\
\hline
\end{tabular}


Table 1. (continued)

\begin{tabular}{|c|c|c|c|c|c|c|c|c|}
\hline Mutation & $\begin{array}{l}\text { Transmis- } \\
\text { sion }\end{array}$ & Brain & Thyroid & Lung & Brain MRI & $\begin{array}{l}\text { N. patients } \\
\text { with brain } \\
\text { MRI }\end{array}$ & $\begin{array}{l}\text { N. patients } \\
\text { with pitui- } \\
\text { tary abnor- } \\
\text { malities }\end{array}$ & References \\
\hline p.W238CfsX9 & De novo & + & + & - & - & 1 & & $\begin{array}{l}\text { Iodice, } 2019 \\
\text { [47] }\end{array}$ \\
\hline §p.W238S & De novo & + & + & - & - & & & $\begin{array}{c}\text { Provenzano, } \\
2016[30]\end{array}$ \\
\hline p.Q240P & De novo & + & + & - & - & 1 & & $\begin{array}{l}\text { Gras, } 2012 \\
\text { [45]; Carrè, } \\
2009[24]\end{array}$ \\
\hline p.R243S & $\mathrm{AD}$ & + & NR & NR & - & 1 & & $\begin{array}{l}\text { Breedveld, } \\
2002[1]\end{array}$ \\
\hline p.R243P & $\mathrm{AD}$ & + & - & - & NR & & & Gras, 2012 [45] \\
\hline p.Y244X & $\mathrm{AD}$ & + & + & - & NR & & & Gras, 2012 [45] \\
\hline p.Q249X & $\mathrm{AD}$ & + & - & - & - & 2 & & $\begin{array}{l}\text { Costa, } 2005 \\
\text { [62] }\end{array}$ \\
\hline p.D252VfsX187 & De novo & + & + & + & - & 1 & & $\begin{array}{l}\text { Parnes, } 2018 \\
\text { [28] }\end{array}$ \\
\hline p.G266del & $\mathrm{AD}$ & + & NR & + & - & 1 & & $\begin{array}{l}\text { Zorzi, } 2008 \\
\text { [63] }\end{array}$ \\
\hline p.G269_271dupGGGa & $?$ & + & - & + & NR & & & $\begin{array}{l}\text { Hamvas, } 2013 \\
\text { [46] }\end{array}$ \\
\hline $\begin{array}{l}\text { p.274_280del7aa and } \\
\text { p.G273fsX152 }\end{array}$ & $?$ & + & + & + & NR & & & $\begin{array}{l}\text { Hamvas, } 2013 \\
\text { [46] }\end{array}$ \\
\hline p.A280fsX161 & $\mathrm{AD}$ & + & + & + & NR & & & $\begin{array}{l}\text { Teissier, } 2012 \\
\text { [48] }\end{array}$ \\
\hline p.P291R & De novo & + & + & - & - & 1 & & $\begin{array}{l}\text { Iodice, } 2019 \\
\text { [47] }\end{array}$ \\
\hline p.L293del & De novo & + & + & + & NR & & & Gras, 2012 [45] \\
\hline p.G303fsX77 & $\mathrm{AD}$ & + & NR & NR & - & 1 & & $\begin{array}{l}\text { Breedveld, } \\
2002[1]\end{array}$ \\
\hline p.A306fsX350 & $\mathrm{AD}$ & + & + & - & - & 2 & & $\begin{array}{l}\text { Moya, } 2006 \\
\text { [37] }\end{array}$ \\
\hline p.Q327fsX121 & De novo & + & + & + & - & 1 & & $\begin{array}{l}\text { Willemsen, } \\
2005 \text { [4] }\end{array}$ \\
\hline p.A327GfsX52 & De novo & + & + & + & NR & & & $\begin{array}{l}\text { Shetty, } 2014 \\
\text { [64] }\end{array}$ \\
\hline p.A329GfsX108 & De novo & + & + & + & NR & & & $\begin{array}{l}\text { Hermanns, } \\
2018 \text { [65] }\end{array}$ \\
\hline p.A333RfsX132 & De novo & + & + & + & - & 1 & & $\begin{array}{l}\text { Tozawa, } 2016 \\
\text { [66] }\end{array}$ \\
\hline p.H349fsX90 & De novo & + & + & + & NR & & & $\begin{array}{l}\text { Hamvas, } 2013 \\
\text { [46] }\end{array}$ \\
\hline p.Q357fsX24 & $\mathrm{AD}$ & + & - & - & - & 3 & & $\begin{array}{l}\text { Mahajnah, } \\
2007 \text { [39] }\end{array}$ \\
\hline p.S366fsX67 & $?$ & - & + & + & NR & & & $\begin{array}{l}\text { Hamvas, } 2013 \\
\text { [46] }\end{array}$ \\
\hline p.T389fsX52 & $?$ & + & + & + & NR & & & $\begin{array}{l}\text { Hamvas, } 2013 \\
\text { [46] }\end{array}$ \\
\hline del 14q13-q21 & De novo & NR & + & + & - & 1 & & $\begin{array}{r}\text { Devriendt, } \\
1998 \text { [67] }\end{array}$ \\
\hline del 14q12-q13.3 & De novo & + & + & + & $-\mathrm{b}$ & 2 & & $\begin{array}{l}\text { Iwatani, } 2000 \\
\text { [68] }\end{array}$ \\
\hline del $141.2 \mathrm{MB}$ & De novo & + & NR & NR & - & 1 & & $\begin{array}{l}\text { Breedveld, } \\
2002[1]\end{array}$ \\
\hline
\end{tabular}


Table 1. (continued)

\begin{tabular}{|c|c|c|c|c|c|c|c|c|c|}
\hline Mutation & $\begin{array}{l}\text { Transmis- } \\
\text { sion }\end{array}$ & Brain & Thyroid & Lung & Brain MRI & $\begin{array}{l}\text { N. patients } \\
\text { with brain } \\
\text { MRI }\end{array}$ & $\begin{array}{l}\text { N. patients } \\
\text { with pitui- } \\
\text { tary abnor- } \\
\text { malities }\end{array}$ & Notes & References \\
\hline del $141.2 \mathrm{MB}$ & $\mathrm{AD}$ & + & + & NR & + & 2 & 1 & $\begin{array}{l}\text { Stalk duplica- } \\
\text { tion }\end{array}$ & $\begin{array}{l}\text { Accornero, } \\
2010 \text { [35] }\end{array}$ \\
\hline del 14q11.2-q13.3 & $?$ & + & + & + & + & 1 & 1 & Cystic mass & $\begin{array}{l}\text { Krude, } 2002 \\
\text { [7] }\end{array}$ \\
\hline del 14q13 & De novo & + & + & + & - & 1 & & & $\begin{array}{l}\text { Carrè, } 2009 \\
\text { [24] }\end{array}$ \\
\hline del $140.9 \mathrm{MB}$ & $\mathrm{AD}$ & + & + & + & - & 3 & & & $\begin{array}{l}\text { Devos, } 2006 \\
\text { [69] }\end{array}$ \\
\hline del 14q12-q13 & De novo & + & + & + & - & 1 & & & $\begin{array}{l}\text { Uematsu, } 2012 \\
\text { [18] }\end{array}$ \\
\hline del 14q13.2-q22.1 & De novo & + & - & - & NR & & & & Gras, 2012 [45] \\
\hline del 14q13.2-q21.2 & De novo & + & + & - & NR & & & & Gras, 2012 [45] \\
\hline del 14q13.3 & De novo & + & + & - & NR & & & & Gras, 2012 [45] \\
\hline del 14q13.1-q21.1 & De novo & + & + & + & NR & & & & $\begin{array}{l}\text { Hamvas, } 2013 \\
\text { [46] }\end{array}$ \\
\hline del $14 q 13.3$ & $?$ & + & + & + & NR & & & & $\begin{array}{l}\text { Hamvas, } 2013 \\
\text { [46] }\end{array}$ \\
\hline del 14q13.3-q21.1 & De novo & + & + & + & NR & & & & $\begin{array}{l}\text { Hamvas, } 2013 \\
\text { [46] }\end{array}$ \\
\hline del 14q13.1-q21.1 & De novo & + & + & + & NR & & & & $\begin{array}{l}\text { Hamvas, } 2013 \\
\text { [46] }\end{array}$ \\
\hline DEL ex 1-2 & $?$ & + & + & + & NR & & & & $\begin{array}{l}\text { Hamvas, } 2013 \\
\text { [46] }\end{array}$ \\
\hline del 14q13.2-q21.1 & De novo & + & + & - & - & 1 & & & Dale, 2012 [70] \\
\hline del $14 q 13.3$ & $\mathrm{AD}$ & + & + & + & NR & & & & $\begin{array}{l}\text { Teissier, } 2012 \\
\text { [48] }\end{array}$ \\
\hline del $14 q 13.3$ & De novo & + & + & - & NR & & & & $\begin{array}{l}\text { Teissier, } 2012 \\
\text { [48] }\end{array}$ \\
\hline del 14q13.2-q21.1 & De novo & + & + & + & - & 1 & & & $\begin{array}{l}\text { Villafuerte, } \\
2018 \text { [71] }\end{array}$ \\
\hline
\end{tabular}

$A D$ autosomal dominant, $N R$ not recorded, ? unknown, $\S$ these mutations, published according to the short NKX2.1 isoform, have been reported to the long isoform ref seq NM_001079668.3, NP_001073136.1.

showed subtle reduced basal ganglia tracer uptake in BHC on nuclear medicine imaging, but concluded that conventional neuroimaging is typically normal [19, 39]. Pituitarysella abnormalities have only been reported in 7 families (7.1\%). A brain MRI scan is reported for 77 patients with NKX2-1 mutations. Furthermore, among these patients, the frequency of pituitary abnormalities reaches $13 \%$, and $26 \%$ in the cases of NKX2-1-related disorders, suggesting that pituitary malformations are present as sign of the disease.

The families reported in the literature showed that the pituitary abnormality is worse in patients with a longer disease duration [8, 9]. Altered pituitary-sella morphology could be an under-recognized phenomenon related to loss of function of NKX2-1 gene. To date, no pituitary abnormalities are reported in the literature in carriers of ADCY5 and PDE10A gene mutations. ADCY5 mutations are known to be more related to atrophy in the frontoparietal cortex and thalamus [72], while PDE10A mutations are more associated with increased signal intensity and atrophy within the striatum (Table 2). [3]

In conclusion, dedicated pituitary imaging should therefore be considered in patients presenting with a clinical phenotype of BHC to guide the diagnosis. In addition, all patients with no brain MRI abnormalities during the first investigations should undergo regular follow-up at a couple of years' intervals. Patients with known abnormalities in the pituitary sella should undergo a routine ophthalmological evaluation including visual fields. Moreover, to rule out pituitary dysfunction, a complete pituitary hormones assay should be routinely performed. We showed the usefulness of the brain MRI with dedicated imaging to the pituitary gland in BHC patients and the value of follow-up imaging in those patients with no changes on the investigation at baseline. The presence of these abnormalities could predict the 
Table 2. MRI characteristics associated with TITF-1, ADCY5, and PDE10A mutations.

\begin{tabular}{lllll}
\hline Gene & Gene product & Inheritance & Age of onset & MRI characteristic features \\
\hline TITF-1/NKX2-1 & Thyroid transcription factor 1 & AD/de novo & Childhood/adulthood & Altered sella turcica morphology \\
ADCY5 & Adenylate cyclase 5 & AD/de novo & Infancy to childhood & Frontoparietal cortex and thalamus atrophy \\
PDE10A & Phosphodiesterase 10A & De novo/AD/AR & Infancy to childhood & $\begin{array}{c}\text { Bilateral striatal hyperintensities and bilat- } \\
\text { eral striatal atrophy }\end{array}$ \\
\hline
\end{tabular}

genetic diagnosis of TTF1-related BHC. Our findings could be useful to improve genetic and neurological counselling of BHC and should be embedded in clinical guidelines.

Supplementary Information The online version contains supplementary material available at https://doi.org/10.1007/s10048-021-00680-3.

Funding PG receives funding from the European Friedreich's Ataxia Consortium for Translational Studies (EFACTS; HEALTHF2-2010-242193; FP7 Grant). ST, PG, MHP, KB, and ID work at University College London Hospitals/University College London which receives a proportion of its funding from the Department of Health's National Institute for Health Research Biomedical Research Centres funding scheme. PG is also supported by North Thames CRN UK.

This project was part of REC 04/Q0505/21.

\section{Declarations}

Competing interests The authors declare no competing interests.

Open Access This article is licensed under a Creative Commons Attribution 4.0 International License, which permits use, sharing, adaptation, distribution and reproduction in any medium or format, as long as you give appropriate credit to the original author(s) and the source, provide a link to the Creative Commons licence, and indicate if changes were made. The images or other third party material in this article are included in the article's Creative Commons licence, unless indicated otherwise in a credit line to the material. If material is not included in the article's Creative Commons licence and your intended use is not permitted by statutory regulation or exceeds the permitted use, you will need to obtain permission directly from the copyright holder. To view a copy of this licence, visit http://creativecommons.org/licenses/by/4.0/.

\section{References}

1. Breedveld GJ, van Dongen JW, Danesino C et al (2002) Mutations in TITF-1 are associated with benign hereditary chorea. Hum Mol Genet 11(8):971-979

2. Mencacci NE, Erro R, Wiethoff S et al (2015) ADCY5 mutations are another cause of benign hereditary chorea. Neurology 85(1):80-88

3. Mencacci NE, Kamsteeg EJ, Nakashima K et al (2016) De novo mutations in PDE10A cause childhood-onset chorea with bilateral striatal lesions. Am J Hum Genet 98(4):763-771

4. Willemsen MA, Breedveld GJ, Wouda S et al (2005) Brain-thyroid-lung syndrome: a patient with a severe multi-system disorder due to a de novo mutation in the thyroid transcription factor 1 gene. Eur J Pediatr 164(1):28-30
5. Salvatore E, Di Maio L, Filla A et al (2010) Benign hereditary chorea: clinical and neuroimaging features in an Italian family. Mov Disord 25(10):1491-1496

6. Bingle CD (1997) Thyroid transcription factor-1. Int J Biochem Cell Biol 29(12):1471-1473

7. Krude H, Schutz B, Biebermann H et al (2002) Choreoathetosis, hypothyroidism, and pulmonary alterations due to human NKX2-1 haploinsufficiency. J Clin Invest 109(4):475-480

8. Veneziano L, Parkinson MH, Mantuano E et al (2014) A novel de novo mutation of the TITF1/NKX2- 1 gene causing ataxia, benign hereditary chorea, hypothyroidism and a pituitary mass in a UK family and review of the literature. Cerebellum 13(5):588-595

9. Treier M, Rosenfeld MG (1996) The hypothalamic-pituitary axis: co-development of two organs. Curr Opin Cell Biol 8(6):833-843

10. Balicza P, Grosz Z, Molnar V et al (2018) NKX2-1 new mutation associated with myoclonus, dystonia, and pituitary involvement. Front Genet 9:335

11. Kimura S, Hara Y, Pineau T et al (1996) The T/ebp null mouse: thyroid-specific enhancer-binding protein is essential for the organogenesis of the thyroid, lung, ventral forebrain, and pituitary. Genes Develop 10(1):60-69

12. Mullis PE (2001) Transcription factors in pituitary development. Mol Cell Endocr 185(1-2):1-16

13. Silberschmidt D, Rodriguez-Mallon A, Mithboakar P et al (2011) In vivo role of different domains and of phosphorylation in the transcription factor Nkx2-1. BMC Dev Biol 11:9

14. Breedveld GJ, Percy AK, MacDonald ME et al (2002) Clinical and genetic heterogeneity in benign hereditary chorea. Neurology 59(4):579-584

15. Gras D, Jonard L, Roze E et al (2012) Benign hereditary chorea: phenotype, prognosis, therapeutic outcome and long term followup in a large series with new mutations in the TITF1/NKX2-1 gene. J Neurol Neurosurg Psychiatry 83(10):956-962

16. Kleiner-Fisman G (2011) Benign hereditary chorea. Handb Clin Neurol 100:199-212

17. Kleiner-Fisman G, Lang AE (2007) Benign hereditary chorea revisited: a journey to understanding. Movement Disorders 22(16):2297-2305

18. Uematsu M, Haginoya K, Kikuchi A et al (2012) Hypoperfusion in caudate nuclei in patients with brain-lung-thyroid syndrome. $\mathrm{J}$ Neurol Sci 315(1-2):77-81

19. Leli DA, Furlow TW Jr, Falgout JC (1984) Benign familial chorea: an association with intellectual impairment. J Neurol Neurosurg Psychiatry 47(5):471-474

20. Glik A, Vuillaume I, Devos D, Inzelberg R (2008) Psychosis, short stature in benign hereditary chorea: a novel thyroid transcription factor-1 mutation. Mov Disord 23(12):1744-1747

21. Fernandez M, Raskind W, Matsushita M et al (2001) Hereditary benign chorea: clinical and genetic features of a distinct disease. Neurology 57(1):106-110

22. Schady W, Meara RJ (1988) Hereditary progressive chorea without dementia. J Neurol Neurosurg Psychiatry 51(2):295-297

23. Schrag A, Quinn NP, Bhatia KP, Marsden CD (2000) Benign hereditary chorea-entity or syndrome? Mov Disord 15(2):280-288 
24. Carre A, Szinnai G, Castanet M et al (2009) Five new TTF1/ NKX2.1 mutations in brain-lung-thyroid syndrome: rescue by PAX8 synergism in one case. Hum Mol Genet 18(12):2266-2276

25. Asmus F, Devlin A, Munz M, Zimprich A, Gasser T, Chinnery PF (2007) Clinical differentiation of genetically proven benign hereditary chorea and myoclonus-dystonia. Mov Disord 22(14):2104-2109

26. Asmus F, Langseth A, Doherty E et al (2009) "Jerky" dystonia in children: spectrum of phenotypes and genetic testing. Mov Disord 24(5):702-709

27. Sussel L, Marin O, Kimura S, Rubenstein JL (1999) Loss of Nkx2.1 homeobox gene function results in a ventral to dorsal molecular respecification within the basal telencephalon: evidence for a transformation of the pallidum into the striatum. Development 126(15):3359-3370

28. Parnes M, Bashir H, Jankovic J (2018) Is benign hereditary chorea really benign? Brain-lung-thyroid syndrome caused by NKX2-1 mutations. Mov Disord Clin Pract 6(1):34-39

29. Patel NJ, Jankovic J (1993) NKX2-1-related disorders. In: Adam MP, Ardinger HH, Pagon RA, et al. (eds) GeneReviews((R)). Seattle.

30. Provenzano C, Zamboni M, Veneziano L et al (2016) Functional characterization of two novel mutations in TTF-1/NKX2.1 homeodomain in patients with benign hereditary chorea. J Neurol Sci 360:78-83

31. Kleiner-Fisman G, Rogaeva E, Halliday W et al (2003) Benign hereditary chorea: clinical, genetic, and pathological findings. Ann Neurol 54(2):244-247

32. Butt SJ, Sousa VH, Fuccillo MV et al (2008) The requirement of Nkx2-1 in the temporal specification of cortical interneuron subtypes. Neuron 59(5):722-732

33. Nobrega-Pereira S, Kessaris N, Du T et al (2008) Postmitotic Nkx2-1 controls the migration of telencephalic interneurons by direct repression of guidance receptors. Neuron 59(5):733-745

34. Lee BJ, Cho GJ, Norgren RB Jr et al (2001) TTF-1, a homeodomain gene required for diencephalic morphogenesis, is postnatally expressed in the neuroendocrine brain in a developmentally regulated and cell- specific fashion. Mol Cell Neurosci 17(1):107-126

35. Accornero S, Danesino C, Bastianello S et al (2010) Duplication of the pituitary stalk in a patient with a heterozygous deletion of chromosome 14 harboring the thyroid transcription factor- 1 gene. J Clin Endocrinol Metab 95(8):3595-3596

36. Steno A, Popp AJ, Wolfsberger S, Belan V, Steno J (2009) Persisting embryonalinfundibular recess. J Neurosurg 110(2):359-362. https://doi.org/10.3171/2008.7.JNS08287

37. Moya CM, Perez de Nanclares G, Castano L et al (2006) Functional study of a novel single deletion in the TITF1/NKX21 homeobox gene that produces congenital hypothyroidism and benign chorea but not pulmonary distress. J Clin Endocrinol Metab 91(5):1832-1841

38. Inzelberg R, Weinberger M, Gak E (2011) Benign hereditary chorea: an update. Parkinsonism Relat Disord 17(5):301-307

39. Mahajnah M, Inbar D, Steinmetz A et al (2007) Benign hereditary chorea: clinical, neuroimaging, and genetic findings. J Child Neurol 22(10):1231-1234

40. McMichael G, Haan E, Gardner A et al (2013) NKX2-1 mutation in a family diagnosed with ataxic dyskinetic cerebral palsy. Eur J Med Genet 56(9):506-509

41. Tubing J, Bohnenpoll J, Spiegler J et al (2018) Methylphenidate can improve chorea in NKX2.1 and ADCY5 mutation-positive patients-a report of two children. Mov Disord Clin Pract 5(3):343-345

42. Nakamura K, Sekijima Y, Nagamatsu K et al (2012) A novel nonsense mutation in the TITF-1 gene in a Japanese family with benign hereditary chorea. J Neurol Sci 313(1-2):189-192
43. Sempere AP, Aparicio S, Mola S, Perez-Tur J (2013) Benign hereditary chorea: clinical features and long-term follow-up in a Spanish family. Parkinsonism Relat Disord 19(3):394-396

44. Pohlenz J, Dumitrescu A, Zundel D et al (2002) Partial deficiency of thyroid transcription factor 1 produces predominantly neurological defects in humans and mice. J Clin Investig 109(4):469-473

45. Gras D, Jonard L, Roze E et al (2012) Benign hereditary chorea: phenotype, prognosis, therapeutic outcome and long term followup in a large series with new mutations in the TITF1/NKX2-1 gene. J Neurol Neurosur Psychiatry 83(10):956-962

46. Hamvas A, Deterding RR, Wert SE et al (2013) Heterogeneous pulmonary phenotypes associated with mutations in the thyroid transcription factor gene NKX2-1. Chest 144(3):794-804

47. Iodice A, Carecchio M, Zorzi G et al (2019) Restless legs syndrome in NKX2-1-related chorea: an expansion of the disease spectrum. Brain Dev 41(3):250-256

48. Teissier R, Guillot L, Carre A et al (2012) Multiplex ligationdependent probe amplification improves the detection rate of NKX2.1 mutations in patients affected by brain-lung-thyroid syndrome. Horm Res Paediatr 77(3):146-151

49. Fons C, Rizzu P, Garcia-Cazorla A et al (2012) TITF-1 gene mutation in a case of sporadic non-progressive chorea. Response to levodopa treatment. Brain Dev 34(3):255-257

50. Konishi T, Kono S, Fujimoto M et al (2013) Benign hereditary chorea: dopaminergic brain imaging in patients with a novel intronic NKX2.1 gene mutation. J Neurol 260(1):207-213

51. Barreiro J, Alonso-Fernandez JR, Castro-Feijoo L et al (2011) Congenital hypothyroidism with neurological and respiratory alterations: a case detected using a variable diagnostic threshold for TSH. J Clin Res Pediatr Endocrinol 3(4):208-211

52. Doyle DA, Gonzalez I, Thomas B, Scavina M (2004) Autosomal dominant transmission of congenital hypothyroidism, neonatal respiratory distress, and ataxia caused by a mutation of NKX2-1. J Pediatr 145(2):190-193

53. Ferrara AM, De Michele G, Salvatore E et al (2008) A novel NKX2.1 mutation in a family with hypothyroidism and benign hereditary chorea. Thyroid 18(9):1005-1009

54. Nagasaki K, Narumi S, Asami T, Kikuchi T, Hasegawa T, Uchiyama M (2008) Mutation of a gene for thyroid transcription factor-1 (TITF1) in a patient with clinical features of resistance to thyrotropin. Endocr J 55(5):875-878

55. Nettore IC, Mirra P, Ferrara AM et al (2013) Identification and functional characterization of a novel mutation in the NKX2-1 gene: comparison with the data in the literature. Thyroid 23(6):675-682

56. Asmus F, Horber V, Pohlenz J et al (2005) A novel TITF-1 mutation causes benign hereditary chorea with response to levodopa. Neurology 64(11):1952-1954

57. Provenzano C, Veneziano L, Appleton R et al (2008) Functional characterization of a novel mutation in TITF-1 in a patient with benign hereditary chorea. J Neurol Sci 264(1-2):56-62

58. Williamson S, Kirkpatrick M, Greene S, Goudie D (2014) A novel mutation of NKX2-1 affecting 2 generations with hypothyroidism and choreoathetosis: part of the spectrum of brain-thyroid-lung syndrome. J Child Neurol 29(5):666-669

59. Koht J, Lostegaard SO, Wedding I et al (2016) Benign hereditary chorea, not only chorea: a family case presentation. Cerebellum Ataxias 3:3

60. Maquet E, Costagliola S, Parma J et al (2009) Lethal respiratory failure and mild primary hypothyroidism in a term girl with a de novo heterozygous mutation in the TITF1/NKX2.1 gene. J Clin Endocrinol Metabol 94(1):197-203

61. Gillett ES, Deutsch GH, Bamshad MJ et al (2013) Novel NKX2.1 mutation associated with hypothyroidism and lethal respiratory failure in a full-term neonate. J Perinatol 33(2):157-160 
62. Costa MC, Costa C, Silva AP et al (2005) Nonsense mutation in TITF1 in a Portuguese family with benign hereditary chorea. Neurogenetics 6(4):209-215

63. Zorzi G, Invernizzi F, Zibordi F et al (2008) Clinical features of a new family with benign hereditary chorea carrying a novel TITF-1 mutation. Mov Disord 23(S1):S1-S422

64. Shetty VB, Kiraly-Borri C, Lamont P et al (2014) NKX2-1 mutations in brain-lung-thyroid syndrome: a case series of four patients. J Pediatr Endocrinol Metab 27(3-4):373-378

65. Hermanns P, Kumorowicz-Czoch M, Grasberger H et al (2018) Novel mutations in the NKX2.1 gene and the PAX8 gene in a boy with brain-lung-thyroid syndrome. Exp Clin Endocrinol Diabetes 126(2):85-90

66. Tozawa T, Yokochi K, Kono S, et al. A video report of brainlung-thyroid syndrome in a Japanese female with a novel frameshift mutation of the NKX2-1 gene. Child Neurol Open 2016;3:2329048X16665012.

67. Devriendt K, Vanhole C, Matthijs G, de Zegher F (1998) Deletion of thyroid transcription factor-1 gene in an infant with neonatal thyroid dysfunction and respiratory failure. N Engl J Med 338(18):1317-1318
68. Iwatani N, Mabe H, Devriendt K et al (2000) Deletion of NKX2.1 gene encoding thyroid transcription factor-1 in two siblings with hypothyroidism and respiratory failure. J Pediatr 137(2):272-276

69. Devos D, Vuillaume I, de Becdelievre A et al (2006) New syndromic form of benign hereditary chorea is associated with a deletion of TITF-1 and PAX-9 contiguous genes. Mov Disord 21(12):2237-2240

70. Dale RC, Grattan-Smith P, Nicholson M, Peters GB (2012) Microdeletions detected using chromosome microarray in children with suspected genetic movement disorders: a single-centre study. Dev Med Child Neurol 54(7):618-623

71. Villafuerte B, Natera-de-Benito D, Gonzalez A et al (2018) The brain-lung-thyroid syndrome (BLTS): a novel deletion in chromosome 14q13.2-q21.1 expands the phenotype to humoral immunodeficiency. Eur J Med Genet. 61(7):393-398

72. Niccolini F, Mencacci NE, Yousaf T et al (2018) PDE10A and ADCY 5 mutations linked to molecularand microstructural basal ganglia pathology. Mov Disord 33(12):1961-1965

Publisher's Note Springer Nature remains neutral with regard to jurisdictional claims in published maps and institutional affiliations. 